\title{
Structural Analysis of Cylinder Head Based on Fluid-solid Coupling Simulation
}

\author{
Yubao Jiang $^{1 \text { a }}$ \\ ${ }^{1}$ School of Mechanical engineering, Beijing Institute of Technology, Beijing 100081,China. \\ ajYB201505@163.com
}

Keywords: cylinder head, finite element, fluid-solid coupling.

\begin{abstract}
For a new diesel engine cylinder head, an assembled finite element model, consisting of cylinder head and cylinder block, is constructed in the present work. By using computational fluid dynamics (CFD) analysis and fluid-solid coupling method, the flow distribution of the water jacket is achieved, as well as the temperature of the cylinder head. Based on the numerical simulation calculation, The results show that the heat transfer efficiency of coolant liquid in the water jacket is uneven, and the temperature of cylinder head is in a reasonable ranges.
\end{abstract}

\section{Introduction}

With the increasing power of engine, the mechanical and thermal loads that the cylinder head beard is sharply increasing. The cylinder head surface directly contacts with high temperature gas, which causes high temperature at the fire surface, as well as stress concentration significantly. The high temperature and stress concentration finally makes the cylinder prone to failure after a long work circulation [1], many researches show that temperature distribution has a great influence on stress distribution. Therefore, it is necessary to investigate cylinder head temperature distribution.

The cylinder head cooling water jacket structure is complex, it is difficult to accurately measure the coolant flow through experiments. Computational Fluid Dynamics (CFD) analysis method is an effective way for the engine cooling system. Fluid-solid coupled heat transfer method can turn the complex outer boundary conditions between the fluid and solid into a relatively simple process, this way not only reduces the number of boundary conditions, but also makes the simulation model closer to the actual working conditions of the engine. Therefore, this fluid-solid coupling method can fully and accurately analyze the cylinder head temperature in the structural design stage.

In the present paper, the finite element method is used to analysis flow distribution characters, as well as cylinder head temperature distribution [2].

\section{The theory of fluid-solid coupling method}

For the question of convective heat transfer problems, the thermal boundary conditions between the fluid and solid can not be given in an advance accurate, but constrained by the interaction between fluid and solid. Fluid-solid coupled heat transfer algorithm by coupling of the two surfaces to achieve solid coupled heat transfer and fluid heat transfer, thus can calculated the required temperature and flow field. The key of the fluid-solid coupled calculations is the heat transfer between fluid and solid [3].

During the calculation process, cooling water flow can be seen as a three-dimensional uncompressed turbulent flow and heat transfers, which satisfy the conservation of mass, momentum and energy conservation laws. Energy conservation equation can be solved solid heat transfer, heat transfer laws follow the Fourier heat conduction law. Both in the form of differential equations, in fully developed turbulent region, may be obtained by standard k- $\varepsilon$ turbulence model equations, which can reflect the impact of the turbulent kinetic energy equation and the turbulent energy dissipation rate equation[4] . 


$$
\begin{aligned}
& \rho \frac{\partial k}{\partial t}=\frac{\partial}{\partial x_{i}}\left[\left(\mu+\frac{\mu_{t}}{\sigma_{k}}\right) \frac{\partial k}{\partial x_{i}}\right]+C_{k}+C_{b}-\rho \varepsilon \\
& \rho \frac{\partial \varepsilon}{\partial t}=\frac{\partial}{\partial x_{i}}\left[\left(\mu+\frac{\mu_{t}}{\sigma_{g}}\right) \frac{\partial k}{\partial x_{i}}\right]+C_{1 g} \frac{\varepsilon}{k}\left(C_{k}+C_{2 g} C_{b}\right)+C_{2 g} \rho \frac{\varepsilon^{2}}{k}
\end{aligned}
$$

In the equation, $\rho$ is density, $\mathrm{k}$ is Turbulent kinetic energy, $\varepsilon$ is Turbulent kinetic energy dissipation rate, $\mu_{t}$ is Turbulent viscosity, $\mu_{t}=\rho C_{\mu} k^{2} / \varepsilon, C_{b}=0, C_{1 g}=1.44, C_{2 g}=1.92, C_{\mu}=0.09$, $\sigma_{k}=1.0, \sigma_{g}=1.3$.

It is mentioned that thermal boundary conditions during heat transfer simulation analysis can not be precisely specified in advance but dynamically determined by the heat exchange process. With the development of science and technology, solving method such as the entire sequence of discrete, whole solving method are investigated. These methods can effective solve the numerical coupled problems. The latter solving way combined the different areas of heat transfer processes as a unified heat transfer process, different regions using a common control equation, and the differ only in diffusion coefficient and generalized source term. By controlling the volume integral method to derive the discrete equation, can satisfy the continuity condition on the interface, eliminating the iterative process between the different areas, so that the calculation time significantly shortened. Therefore, this method becomes an effective way to solve the coupled heat transfer problems. In the fluid-solid coupling simulation process, the finite element software provides a standard wall function treatment of the boundary layer flow and heat transfer boundary layer.

\section{Fluid-solid coupling analysis}

\subsection{CFD analysis.}

The model of cylinder head used in this study cover on a V6 engine, this cylinder head using high-temperature drilling cooling technology, which can reduce the volume of the cooling system. At the same time, the character of the cylinder head is nose bridge area prone to stress concentration. The model of single body-head water jacket is shown in Fig 1. According to the design requirements, each cylinder has an intake, for V6 engines, each row of three-cylinder has a common outlet.

AVL-FIRE software is used to conduct an overall CFD flow field analysis. Take the first cylinder as research subjects, in order to obtain single cylinders boundary conditions, the first step is simulate the whole single row of the body -head water jacket water flow. At the beginning of the calculation, the input parameters are inlet mass flow and outlet pressure. Fig 1 shows the model of water chamber.

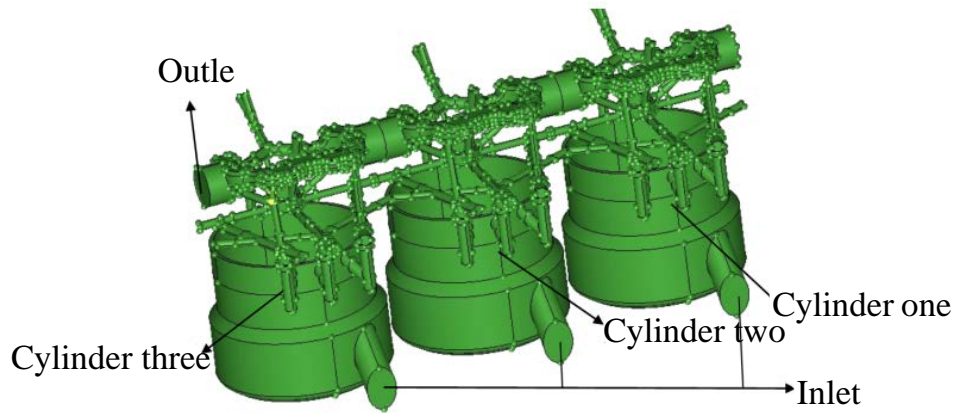

Fig 1 The model of water chamber

Fig 2 shows the results of the water flow field. It can be seen from Figure 2(a) that cylinder one and two water chamber's fluid pressure are significantly higher than the cylinder three, cylinder one's mean pressure larger than cylinder three, which value is $13000 \mathrm{~Pa}$, and the maximum pressure loss reached $62253 \mathrm{~Pa}$. As can be seen from Figure 2(b), from cylinder one to cylinder three, the cylinder head cooling water flow rate increases. The cylinder three's water jacket cooling water flow rate is higher than the average of $3 \mathrm{~m} / \mathrm{s}$, and the cylinder one's water average velocity less than $1 \mathrm{~m} / \mathrm{s}$.

The simulation results showed that each cylinder head cooling water chamber takes series connection, which lead to each chamber's pressure, velocity distribution is uneven. The water 
chamber which close to the outlet, the flow rate is higher than others, As well as the low water inlet flow rate causes cylinder three's cooling uneven. This water jacket did not achieve a good cooling effect.
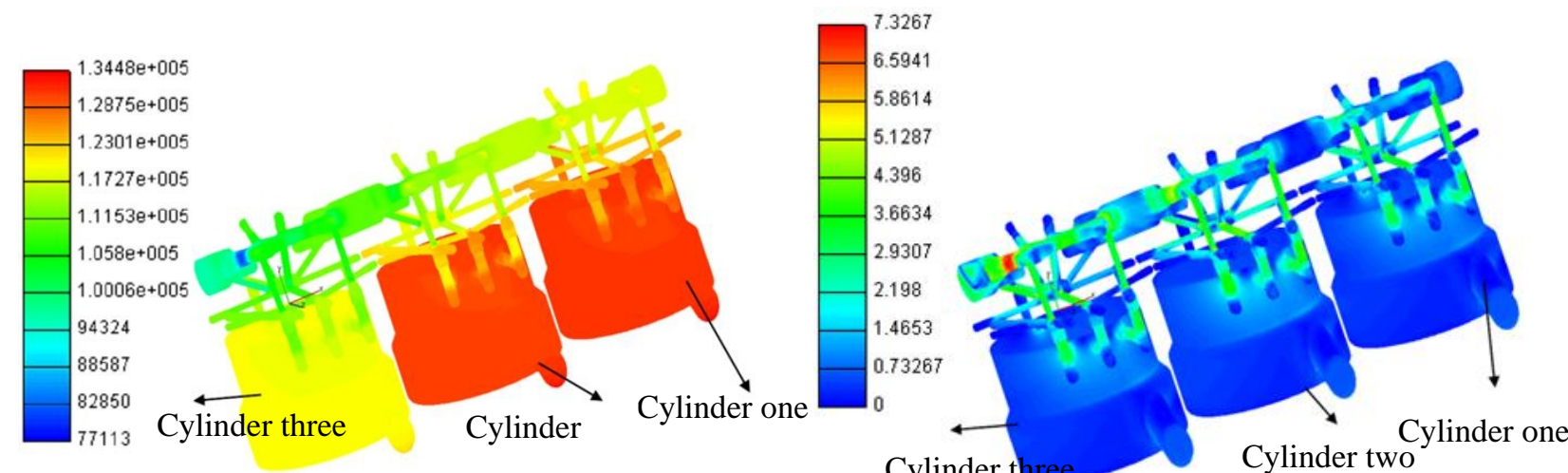

(a) Absolute pressure distribution of water chamber

(b) Velocity distribution of water chamber

Fig.2 CFD simulation results

\subsection{Single cylinder water flow analysis}

Above the cooling water flow field of the chamber as a whole analyzed, the results show that the cooling efficiency of each cylinder is uneven. This part will check the key parts of a single-cylinder cooling effect. Fig 3(a) is a single cylinder body-head water jacket model. Fig 3(b) is the single-cylinder water chamber coolant velocity field. The vertical cooling pipe that connect the bottom of the chamber and the upper pipe has high flow rate. The vertical cooling pipe located at the exhaust side of the cylinder head, which has a good cooling effect on cylinder's exhaust side. But for the study of the cylinder head, because the fire surface withstands high temperatures, the most important parts of the cylinder head at fire surface is nose bridge regions, which does not be cooled efficiently. Fig 3(c) shows the coolant flow rate at nose bridge area. The simulation results indicate a problem that the water jacket that taking drill cooling design form does not make fire surface temperature well cooled. Besides, the thickness of center ring only $1.58 \mathrm{~mm}$, that means water circulation area vary widely, which lead to a large loss of water pressure, and water flow is not smooth.

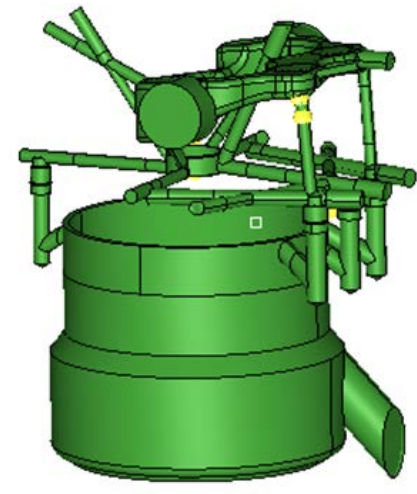

(a) Single cylinder water jacket model

$$
\begin{array}{|l|l|}
\hline & 5.1864 \\
4.6678 \\
4.1491 \\
3.6305 \\
3.1118 \\
2.5932 \\
2.0746 \\
1.5559 \\
1.0373 \\
0.51864 \\
0
\end{array}
$$

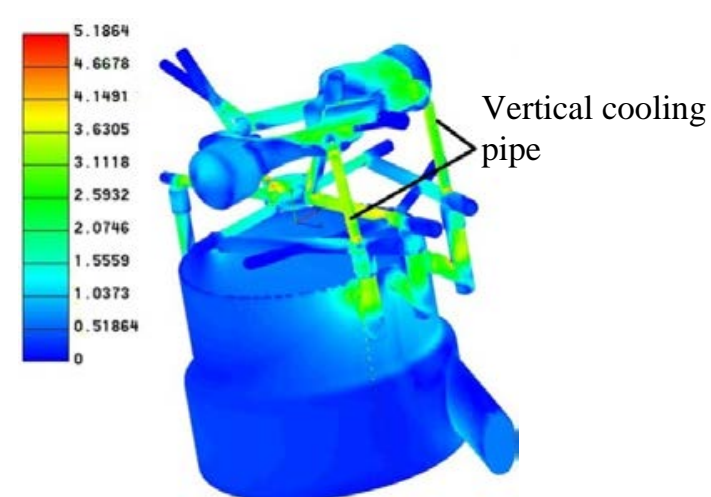

(b) Single cylinder water velocity distribution

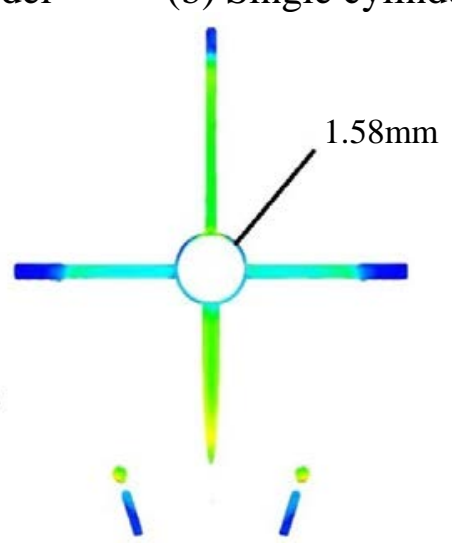


(c) The key regions water velocity

Fig 3 Single cylinders model and simulation results

Depending on the previous analysis of the flow field in the cylinder head, cooling system is carried out in this section to calculate the cylinder head temperature distribution. In order to achieve fluid and solid coupling simulation calculation, the first step is acquire water jacket's thermal boundary condition and mapped it to the water chamber surface mesh. After the initial mapping of thermal boundary conditions and coupling calculation, it will be able to get the cylinder head temperature distribution in this state. During the first time of CFD calculations, because the water chamber wall's temperature distribution can not be accurately defined. Therefore, it is inevitably exists some errors. After several iterations, compared two times calculation results, until the calculation error within the allowable ranges[5]. Depending on the CFD analysis results and material's thermal conductivity, finally, cylinder head temperature distribution can be obtained. Fig 4 shows the temperature distribution, it can be seen that the maximum temperature is about $405{ }^{\circ} \mathrm{C}$, appeared at the center of the nose bridge area between the exhaust valve.

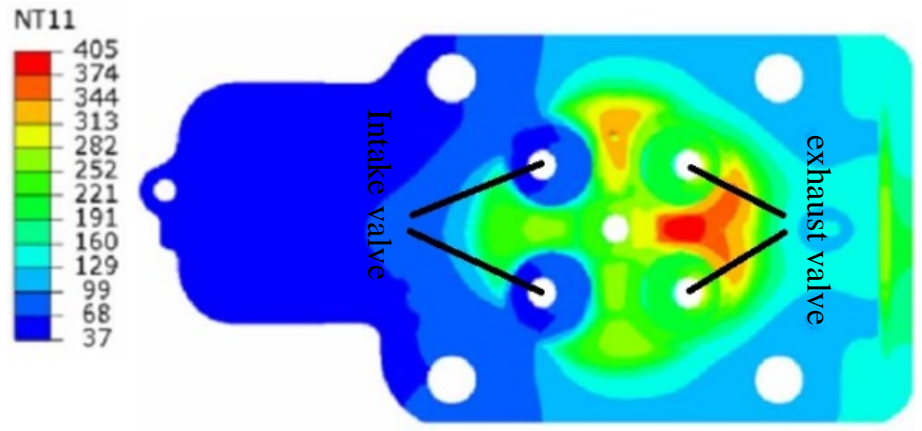

Fig 4 Temperature distribution of cylinder head

Tab 1 Material's thermal conductivity

\begin{tabular}{lccccccc}
\hline Temperature $\left({ }^{\circ} \mathrm{C}\right)$ & 25 & 100 & 200 & 300 & 400 & 500 & 600 \\
\hline Thermal conductivity $(\mathrm{W} /(\mathrm{m} \cdot \mathrm{K}))$ & 29.9 & 28.9 & 31.5 & 33.3 & 32.3 & 31.3 & 28.8 \\
\hline
\end{tabular}

\section{Summary}

(1)The cylinder head's dynamics flow simulation results show that each chamber's pressure, water velocity is uneven, which also finally lead to different cooling effect.

(2) By fluid-solid coupling analysis, temperature distribution of the cylinder head is obtained, as well as the maximum surface temperature of the cylinder head located at nose bridge area which between the exhaust valves.

(3)The simulation results show that the maximum surface temperature reached $405{ }^{\circ} \mathrm{C}$, although the temperature is high, but still in the range of material can beard.

\section{References}

[1]. Espadafor F, Villanueva J, García M, et al. Analysis of a diesel generator cylinder failure[J]. Engineering Failure Analysis,2010, 17:913-925.

[2]. Stefano F, Matteo G. Multiphase CFD-CHT optimization of the cooling jacket and FEM analysis of the engineheadof a V6 diesel engine[J]. Applied Thermal Engineering, 2013, 52:293-303.

[3]. Deng B L, Liu J P, Feng R H. An Analysis on the Temperature Field of Cylinder Head Based on Fluid-Solid Coupling[J]. Automotive Engineering,2012,34(3):202-206.

[4]. Li Y, Yu X L, Chen H Y. 3-DSimulation of Steady Heat Transfer of Fluid-Solid Coupled System in Engine Coolant System[J].Transactions of CSICE, 2007, 25(3):252-257. 
[5]. Zhang Q, Zuo Z X, Liu J X. Failure analysis of diesel engine cylinder head based on finite element method[J]. Engineering Failure Analysis, 2013,34:51-58. 